\title{
Lucia Vedovi
}

\section{L'espace letterario in Stella mattutina di Ada Negri}

\begin{abstract}
Nel 1920 Ada Negri annuncia la pubblicazione di Stella mattutina con le seguenti parole: «Stella mattutina è la storia interiore della mia vita dai sette ai diciassette anni, in terza persona. Vi ho scolpito la figura di mia madre.» Avvalendosi delle conclusioni teoriche sul semeotico formulate da Julia Kristeva in Revolution in Poetic Language (1984) e in Language - the Unknown: an Initiation into Linguistics (1989), nonché del concetto di espace pittorico delineato da Johanna Drucker in Theorizing Modernism: Visual Art and the Critical Tradition (1994), il presente saggio intende esaminare come Negri ricostruisca la storia del nucleo familiare tutto al femminile in cui crebbe e si formò attraverso l'impiego di un originale impasto linguistico che fonde prosa e poesia. Questo peculiare stile permette alla scrittrice di sfondare i tradizionali concetti di spazio e tempo e creare un espace lirico nel quale ella, da un lato, rappresenta la storia della classe operaia italiana alla fine del diciannovesimo secolo; dall'altro, rivendica il ruolo delle scrittrici nell'Italia pre-fascista, dominata dai diktat di una società essenzialmente patriarcale. Inoltre si dimostra che, travalicando i confini di quello che Gregory Castle in Reading the Modernist Bildungsroman definisce "a genre in crisis», il concetto di un espace letterario virtuale, che caratterizza la modernità e il modernismo di questo romanzo di formazione, ha la funzione di creare uno spazio 'altro', una patria riscoperta e, in ultima istanza, «una stanza tutta per sé» nell'opera di una scrittrice di spicco qual è Ada Negri, forse ancora non del tutto consacrata dal canone ufficiale.
\end{abstract}

Nella prefazione all'edizione del 2008 di Stella mattutina di Ada Negri, Gianguido Scalfi, nipote della scrittrice lodigiana, afferma: «Non è un romanzo autobiografico. La componente poetica prevale. Mia nonna mi disse che molte di queste pagine furono scritte da lei, al mattino, al risvegliarsi, quasi in sogno.» ${ }^{1}$ Scalfi rileva, inoltre, due aspetti cruciali nell'opera che, sostiene, non vennero colti dalla critica per lungo tempo: da un lato, la sorprendente modernità dello stile «scarno, semplice, scorrevole» che la distanziava di netto dai romanzi d'appendice; dall'altro, una presa di coscienza profonda dei temi sociali che portarono l'autrice ad una simbiotica identificazione con l'“altro”. Muovendo da questi presupposti, il presente saggio intende dimostrare come i legami tra la componente poetica, la modernità dello stile e i temi sociali in Stella mattutina prendano forma parallelamente e si alimentino tutti all'interno di un peculiare spazio letterario modernista che definiremo espace, avvalendoci delle riflessioni teoriche sul concetto di espace pittorico formulate da Johanna Drucker in

The e-journal «altrelettere» is hosted at the URL: http://www.altrelettere.uzh.ch, in accordance with the Open Access Policy of the University of Zurich. Please cite this article as follows: Lucia VEDOVI, L'espace' letterario in "Stella mattutina" di Ada Negri, in «altrelettere», 4.2.2014, DOI: 10.5903/al_uzh-16.

(C) This article is licensed under a Creative Commons Attribution 2.5. Switzerland (CC BY-NC-ND 2.5). Please read the license terms on the website: http://creativecommons.org/licenses/by-nc-nd/2.5/ch/deed.en 
Theorizing Modernism: Visual Art and the Critical Tradition (1994). Il fenomeno della creazione di uno 'spazio altro' all'interno del tessuto compositivo di un'opera pittorica sembra individuabile anche in ambito letterario e, nel caso specifico di Negri, assolverebbe a una duplice funzione: in prima istanza, le permette di trasformare la propria vicenda di bambina ribelle ed adolescente appassionata di letteratura in un vero e proprio récit, tipizzando la situazione di precarietà della donna italiana d'umili origini alla fine dell'Ottocento; dall'altro, le consente di rivendicare uno spazio letterario tutto al femminile in un'Italia prefascista essenzialmente dominata dai diktat di una società patriarcale. L’innovativo amalgama prosastico, imbevuto di lirismo, verrà analizzato avvalendosi delle teorie semeotiche di Julia Kristeva esposte in Revolution in Poetic Language del 1984 e in Language - the Unknown: an Initiation into Linguistics del 1989.

Il lavoro di Johanna Drucker prende in esame la rivoluzione che investe le arti figurative in Europa nel passaggio dal movimento impressionista alla nascita dell'Espressionismo e che culminerà con la nascita del Cubismo. La studiosa americana evidenzia come, per la prima volta, in pittura si assista non tanto alla rappresentazione della realtà, quanto alla presentazione di una realtà altra rispetto a quella percepita dall'occhio nudo. Se l'intento della scuola impressionista di Monet era stato quello di mettere su tela la rarefazione del soggetto rappresentato attraverso il filtro degli agenti atmosferici, e se, di pari passo, la corrente pointillista aveva puntato alla scomposizione delle immagini in atomi di colore, a partire dalla pittura post-impressionista di Cézanne si registra un fenomeno del tutto nuovo: la ri-produzione di soggetti naturalmente visibili all'occhio, o comunque registrabili in modo scientifico e quindi attinenti alla realtà esterna, lascia il posto alla produzione di immagini che attengono alla realtà interiore dell'individuo. In altre parole, a venir ritratto è ciò che sfugge all'eye perché pertinente all' $I$, e che non è fisicamente presente nella dimensione dello spazio esterno al dipinto bensì nell'espace subcosciente dello spettatore. 
The focus on the space represented was gradually reformulated to emphasize the increasing conceptual autonomy of representational space, or espace. [...] [A] painter whose work offers clear evidence of this transition, and its characterization toward the insistent autonomy of the work of the Cubists, [is] Paul Cézanne. ${ }^{2}$

Secondo Drucker questa nuova figura di artista non guarderebbe più al soggetto da riprodurre con intento imitativo bensì al fine di ritrarne il nonvisibile, l'interiorità, il sub-conscio; di conseguenza anche il rapporto dello spettatore con il soggetto varierebbe irreversibilmente, in quanto verrebbe a mancare una immediata identificazione tra le due parti.

For [this] artist, the "truth in seeing" which [he] struggles to define through [his] paintings is always evaluated according to what occurs within the limited parameters of the canvas in its capacity to produce a visual truth. For Cézanne that truth is grounded in nature as an ideal model [...] For [him], painting became a space, espace, a laboratory of productive investigation and experiment, which had less and less relation to the literal space of modern life. (DRUCKER 1994, 38)

Di fatto, a partire dalle innovative tecniche formulate dall'artista provenzale, il processo spazio-retina-cervello viene surclassato dalla sequenza espace-retinasubconscio. Il soggetto stesso del dipinto funzionerebbe, quindi, quale mero escamotage perché l'espace dell'artista e l'espace dello spettatore possano entrare in contatto:

for Cézanne painting is a form of knowing, the image is knowledge: one in which the espace of the canvas can construct and reveal through visual means something which is not ever apparent merely to the eye [...] The concept of espace thus serves to expand the notion of the space of modernity beyond the literal, into the conceptual domain in which knowledge and experience of modern life may be presented and understood through visual means. (DRUCKER 1994, 44-46).

In campo più strettamente letterario, Drucker applica le stesse speculazioni alla nota figura del flâneur baudelairiano il cui ruolo oscilla tra quello di spettatore e di artista nel pre-moderno contesto urbano di fine Ottocento. Anticipando numerosi studi che avrebbero indagato la parzialità di una prospettiva esclusivamente maschile come quella baudelairiana ${ }^{3}$, la teorica americana riconosce che il punto di vista della donna è puntualmente escluso dalle riflessioni dell'autore francese; ella è eventualmente presa in considerazione sempre e solo come 'osservata' e mai come 'osservatrice'. 
The roles of flâneur as spectator and spectator as artist proposed by Baudelaire were decidedly biased toward a male and bourgeois characterization. [...] Baudelaire's description of the flâneur's movement was immediate, present, and vivid [...] By contrast, the roles [Baudelaire] presented for women were all objectified, distanced, observed through and across a space, physical/literal and social/metaphoric. (DRUCKER 1994, 14)

In ambito italiano, le coordinate spaziali descritte all'interno del romanzo Stella mattutina sembrano presentare delle evidenti affinità con il concetto di espace appena esposto. Benché generalmente catalogato con la dicitura di romanzo di formazione o, più genericamente, autobiografico, ed allineato alle produzioni più o meno contemporanee di Sibilla Aleramo e Grazia Deledda, nell'opera in questione si riscontrano dei tratti strutturali che la contraddistinguono e distanziano dal genere Bildungsroman tout court. Non a caso, il 18 dicembre del 1920, Negri commenta l'uscita dell'opera con le seguenti parole: «Stella mattutina è la storia interiore della mia vita dai sette ai diciassette anni, in terza persona. Vi ho scolpito la figura di mia madre»4. L'enfasi della dichiarazione è posta tanto sul valore contenutistico del romanzo quanto, se non maggiormente, su quello strutturale. L’incipit del romanzo, «Io vedo - nel tempo - una bambina.» (SM 13), sembra confermare tale ipotesi. L' «Io vedo» è indubbiamente la voce dell'autrice/narratrice che - usando la prima persona in quest'unica occasione all'interno dell'opera - dichiara di guardare alla bambina Dinin, protagonista della narrazione, attraverso il tempo del romanzo, racchiuso all'interno dei due trattini. Il tempo diventa così spazio fisico, un vero e proprio palcoscenico teatrale che la narratrice allestisce per mettere in scena la sua storia. Subito dopo ci viene detto che «[la bambina] non potrà, più tardi, aver memoria del proprio viso di allora.» (SM 13) È evidente che Negri postuli come presupposto al suo lavoro letterario l’incapacità di far fede sui propri ricordi d’infanzia e, va da sé, l’impossibilità di ricostruire la propria autobiografia senza in qualche modo falsarla, o, per meglio dire, romanzarla. Non a caso le vicissitudini della protagonista e della sua disagiata famiglia si mescolano, nelle prime pagine, alla figura della «meravigliosa contralto» Giuditta Grisi, la cui favolosa vita porta la bambina a pensare «Anch'io andrò sul teatro» (SM 15). Il suo teatro, quello sul quale le viene data la possibilità di esibirsi per noi 
lettori/spettatori, altro non è se non il libro che abbiamo in mano, l'opera scritta ed allestita per lei dall'autrice. Grazie a questa messinscena letteraria, la bambina può indossare gli abiti di Grisi e far sì che «Le flosce vesti pendenti si riempi[a]no, quando vuole, di ossa e di carne: spunt[i]n da esse mani e teste: voci ne esc[a]no: un moto illusorio le anima. Giuditta Grisi» - e la bambina con lei «canta. Il pubblico immaginario» - e noi lettori con esso - «applaude». (SM 16). Ed è qui significativo notare il reiterato utilizzo dei due punti, come a rendere ecfrasticamente l'allestimento della scena e l'ingresso sul palco della protagonista davanti ai nostri occhi. Tuttavia, le vesti che la piccola sceglie di indossare sono quelle di mamma Vittoria, operaia, e di nonna Peppina, custode del palazzo Cingia-Barni - dove la poetessa visse veramente durante l'infanzia - che ben poco hanno da spartire con l'esistenza agiata della soprano. In effetti, in tutto l'opus letterario di Negri la denuncia sociale spicca come tema portante e trait d'union tra la produzione poetica e quella prosastica. Come giustamente rileva Scalfi, il coro di personaggi che fanno da sfondo alle vicende principali di Stella mattutina, composto di umili operai, ciabattini, falegnami, ecc. e le descrizioni che la protagonista ne fa, riecheggiano molte delle liriche delle raccolte precedenti al romanzo, come il seguente passo tratto da I grandi del 1895

sono/gli affamati, gli oppressi, i venerandi,/che per altri fiorir vider la spica,/e non hanno rubato:/che bevver fiele e lacrime, vilmente/frustati in pieno viso/da l'ingiustizia cieca e prepotente/e pur non hanno ucciso:/che passaron fra i geli e le tempeste/in basso, ne l'oblio/senza sol, senza pane, senza veste,/ed han creduto in Dio. 5

Significativi anche i versi di Senza nome: «Io non ho nome. Io sono la rozza figlia/dell'umile stamberga;/Plebe triste e dannata è la mia famiglia». 6

Le riflessioni di Dinin, imbevute di risentimento per quei padroni ricchi e nobili che l'accusano di rubare e ai quali malvolentieri si presta ad aprire il cancello della lussuosa dimora, sono l'eredità della lirica passata e l'embrione della futura scritta «col sangue dei fratelli caduti e coi singulti dei ribelli» (GORINI SANTOLI 1995, 24) da parte di una poetessa che porta avanti una crociata a favore delle classi umili e costantemente sfruttate, con le quali entra in contatto anche attraverso il suo lavoro di insegnante. Gorini Santoli parla di «dolore cocente, 
quasi intriso di rabbia per la condizione operaia di cui è vittima la sua amatissima mamma; cui si aggiunge [...] la constatazione della miseria dei contadini, dei boscaioli e dei battellieri, e gli incontri con certe povere mamme di suoi allievi con il giallore della pellagra in faccia» (GoRINI SANTOLI 1995, 31).7 Alla forte denuncia sociale fa da contraltare una profonda amarezza per un mancato riconoscimento quale scrittrice nel contesto letterario italiano di inizio Novecento. Non di rado Negri nutrì forti dubbi nei confronti delle proprie creazioni, tanto da dichiarare nel 1935: "Io morirò rimproverandomi ciò che in arte non ho dato, e forse avrei, volendo dare» e, nel 1940, a cinque anni dalla morte: "Altre liriche ho, tutte di non più di una pagina. Vorrei fare un libro così. Ma non sono che al principio. E la vita precipita». ${ }^{8}$ Ciò che sembra dilaniare la scrittrice è l'idea di non essere riuscita a scrivere il suo libro perfetto, l'opera che racchiudesse, in un calibrato amalgama letterario, attivismo sociale e velleità letterarie. Eppure a noi pare che mai tanto magistralmente quanto in Stella mattutina Negri compia due atti rivoluzionari: in primo luogo, ella trova il giusto impasto tra lirica e prosa - contravvenendo ai dettami crociani secondo i quali la poesia era territorio esclusivamente maschile, per cui le donne avrebbero dovuto limitarsi all'espressione prosastica - quindi, in seno a tale impasto, crea lo spazio rappresentativo - appunto, l'espace - che rende giustizia alle voci messe a tacere dalla storia italiana ufficiale: nella fattispecie, gli esponenti delle classi sociali più basse e le scrittrici. Quello che si potrebbe woolfianamente definire un «espace of one's own» apre una strada letteraria nuova sulla cui scia si immetteranno altri nomi altisonanti della letteratura italiana femminile del Novecento, quali l'Anna Banti di Artemisia (1947) o l'Ortese di Il Porto di Toledo (1975). Le autrici/narratrici di queste opere scelgono di rinunciare ai parametri del romanzo autobiografico tradizionale - i cui protagonisti sono necessariamente portati a mentire nel raccontare di sé, come ci dimostra il capolavoro di Elsa Morante, Menzogna e Sortilegio (1948) - e decidono di decostruire e ricostruire i propri personaggi attraverso una memoria creativa che è commistione di vero e verosimile, di ricordi e d'invenzione, di tempo memorizzato e di tempo proustianamente recuperato. 
Nel caso specifico di Stella mattutina lo sfondamento dello spazio tradizionale a favore dell'espace del Bildungsroman modernista è innescato da una continua oscillazione tra espressione prosastica ed eco lirica. Il seguente passaggio esemplifica il costante slittamento tra lo spazio della rappresentazione del vero autobiografico e l'espace della presentazione del verosimile autobiografico:

Fra l'invetriata a smeriglio verso la strada e le vaste intelaiature a cristalli verso il porticato, la portineria giace in un chiarore pallidissimo d'alba. In quella spettrale bianchezza, la nonna, immobile sulla poltrona, pare una figura di pietra. Neve sopra neve cade in giardino, incappuccia alberi e cespugli, copre le panche di soffici cuscini quasi azzurri a fissarli, ricama cornicioni e balaustri, vuol dire alla fanciulletta tante cose, che questa cerca di comprendere e ancora non può. È una specie di lungo discorso in una lingua ignota, pieno di pause misteriose, dolcissimo. [...] ella pensa di essere rimasta sola nel mondo. Non più padroni, non più scuola, più nulla: nemmeno la madre. Le si dilata l'anima: le diviene leggera leggera: aderisce alla neve, si fa un fiocco di neve, scompare nel bianco. (SM 22).

Si osservi come, in questo passaggio, il testo scivola dalla descrizione delle vicende che ripercorrono l'evoluzione del personaggio dell'autrice/narratrice e si dilati fino ad accogliere il lettore nel proprio espace lirico in cui emerge l'“altra" realtà narrativa, quella interiorizzata.

In altri casi, la narrazione viene addirttura interrotta per far spazio a brevissimi passaggi la cui struttura s'impregna di un evidente lirismo: «Canto fermo su accompagnamento d'organo, in una chiesa nuda, piena di poveri che ascoltano la messa: tali le loro vite. Ma una stonatura vi stride ogni tanto; e non si sa se sghignazzi o se pianga, spezzando la grave armonia dell'insieme: Nanni» (SM 35-6). Il prevalere della sintassi nominale e la postposizione del soggetto alla fine della frase creano un ritmo discendente che fa scivolare il lettore in uno spazio intimo, segreto, subcosciente.

Gorini Santoli giustamente rileva come

[Negri abbia] unito vita e arte in un nodo assai raro, strettissimo e inscindibile, onde percorrere il suo cammino artistico comporta affiancarne un altro, quello della sua vita, da compiersi con estrema attenzione: l'attenzione con cui ella, ignara di Freud e Lacan, ha guardato entro e fuori se stessa, consapevole di quanto la vita dell'uomo sia debitrice ai suoi primi anni. (GORINI SANTOLI 1995, 25)

Altrettanto convincente risulta la tesi di Giorgio Rimondi secondo cui «nella misura in cui [...] il testo narrativo funziona come 'una macchina da far vedere', il 
passaggio dalle arti visive alla letteratura [si configura] come semplice traslazione di concetti entro il [campo scopico] che nel pensiero di Lacan è fondamentale per la comprensione dello statuto del soggetto».9

Ciò nonostante, il modo in cui la narratrice di Stella mattutina guarda alla bambina che è stata e ne ritrae sia lo spazio fisico in cui si muove, sia il suo espace interiore, sembra mettere in luce aspetti della sua psicologia di scrittrice che rimandano sostanzialmente alla semeotica kristeviana. La costante fluttuazione tra la langue saussuriana della prosa tradizionale che ci racconta la bambina Dinin e il lirismo delle riflessioni dell'Altra, ossia della poetessa in nuce che la figlia di Vittoria sente emergere prepotentemente dentro di sé - e alla quale non riesce a dare un nome/significante - sembrano anticipare l'idea di chant di cui Julia Kristeva parla nel capitolo Language and Languages in Language - the Unknown, in riferimento alla poesia di Mallarmé:

In certain cases, the search for the autonomy of the signifier, impregnated with a signified that seems superimposed on the signified of the explicit message, is taken so far that the poetic text constitutes a new language. [...] Mallarmé wrote in order to create through French such an other language. [...] The new language, to be constructed, traverses the natural language and its structures, or transposes it. [...] It decenters the apparent structure of communication and produces a supplementary meaning, a chant. ${ }^{10}$

Nell'attraversare le strutture naturali, o per meglio dire simboliche, del linguaggio codificato, il nuovo linguaggio, fatto di prosa liricamente evocativa, crea l'espace all'interno del quale la protagonista diventa tante protagoniste in una. L“io” del sintagma iniziale vede - 'per-see-ves' - se stesso attraverso questo espace e ci racconta la bambina che la narratrice è stata: la nipote di Peppina; la figlia di Vittoria; Dinin; infine l'Altra, che le contiene tutte. Di conseguenza, il romanzo di formazione si scompone e ricompone in un'installazione di prospettive multiple e di passaggi di parola talvolta così disorientanti da non poter stabilire con assoluta chiarezza chi racconti e chi ascolti La storia di donna Augusta e La storia di donna Teodosia, i due brevi racconti narrati da mamma Vittoria che interrompono bruscamente la trama principale del romanzo. Anna Folli descrive la narratrice come 
Sempre in bilico tra vedere e sentire, solo raramente ha coscienza di sé come totalità. Dice Io e tuttavia cerca di eludere la legge dell'autobiografismo che vincola al vero. Si rifugia nel racconto e nella descrizione, nello pseudonimo, nella terza persona, perfino si fa schermo di un personaggio pur di alleggerire lo schiacciamento dei fantasmi della narrazione, che vive poi come proiezioni della propria molteplicità. (FOLLI 2000, 117)

Le due storie sopra menzionate concorrono a ribadire il fatto che Negri si relaziona con la se stessa protagonista del romanzo così come mamma Vittoria si rapporta con i personaggi Augusta e Teodosia; in altre parole, l'autrice/narratrice dipinge un'immagine falsata di sé proprio come Vittoria fa con le eroine dei suoi racconti.

Può essere interessante, a questo punto, prendere in esame quanto postulato da Danielle Hipkins in Contemporary Women Writers and Traces of the Fantastic - The Creation of Literary Space. Secondo la studiosa, «in fantastic fiction women writers find opportunities to transgress self-consciously many textual boundaries, by re-forming language, re-shape notions of self and reality [...] the fantastic is used to shift reader and writer into a new relationship with the text and the real».11 Il fantastico sarebbe quindi il territorio ideale per la costante oscillazione tra il simbolico e il semiotico e la rinegoziazione dei termini di rapporto tra scrittore e lettore secondo i dettami di Roland Barthes. Per il linguista francese, come ci ricorda Liz Stanley, «'the self who writes' no more has direct and unproblematic access to 'the self who was', than does the reader». ${ }^{2}$

Una teoria analoga si riscontra nelle riflessioni di Monica Farnetti in Geografia, Storia e Poetiche del Fantastico, in cui la scrittrice delinea il genere fantastico come «indicante a un tempo, e non senza implicazioni, nella letteratura, un massimo di verità e un massimo di finzione [...] [ciò ci permette di] distinguere se non di opporre il vedere ciò che 'c'è', è fisicamente copresente al soggetto nell'atto del suo percepire, rispetto a ciò che 'non c'è' ma che, al livello di altri sistemi produttivi di immagini, risulta presente e in ogni caso 'visibile'». ${ }^{13}$

Benché Stella mattutina non appartenga specificamente alla categoria del fantastico, sembra che vi si possano rintracciare gli stessi presupposti. Nell'atto di scolpire la figura della madre, e nel frantumare e ri-assemblare la sua stessa figura di bambina e poetessa, Negri coinvolge anche il lettore nella volontà di passare in consegna un messaggio al contempo letterario e sociale. Infatti, al 
termine di La storia di donna Augusta, la narratrice riprende la parola per trasformare il grido finale della povera contessina morta per amore in un grido che coinvolga tutti i sofferenti:

Gemito che viene dal mistero iniziale degli esseri, e da un altro mistero, la morte, è inghiottito: triplice gemito che non chiede aiuto, ma solo cerca di liberare la carne che soffre: la figlia di Vittoria se lo ritroverà nel cuore, se lo ritroverà sulla bocca, nelle ore di angoscia. Con la vita, la madre le ha dato anche quel gemito: glielo ha messo nelle radici dell'anima. Per se stessa, e per gli altri: per i propri dolori, e per tutti i dolori umani; di supremo spasimo, ma anche di supremo amore: - Ahi, ahi, ahi. (SM 69)

Da questo grido di sofferenza - che è anche protesta per le ingiustizie subite dall'operaia Vittoria - nasce la lirica Mani nellingranaggio, che la figlia di Vittoria «scrive [...] per bollare a sangue un'ingiustizia: compie un atto di necessità». (SM 82) E nel farlo, si lascia prendere la mano - anche la sua, in fondo, è una mano nell’ingranaggio, quello letterario - facendosi «trascinare ad una deformazione del vero» (SM 83). Questo breve passaggio risuona come una dichiarazione da parte dell'autrice/narratrice della volontà di travalicare le costrizioni della rappresentazione del vero per attingere alla dimensione del verosimile. Riallacciandosi alla suddetta teoria barthiana, si riporta l'analisi di Liz Stanley:

Making Barthes' apparently simple distinction between the self who writes, the self who was, and the self who is, immediately challenges the central myth of auto/biographical writing, the myth of a single, coherent, stable and gradually unfolding inner and indubitably real essential self. Auto/biography smooths out of sight doubts as to the nature of the self - or at least those selves who write or have auto/biographies written about them, if not the selves of common readers - by treating its reality as an a priori truth. (STANLEY 1992, 133)

L'autrice Ada Negri fa appello alla narratrice perché chiami in causa i suoi lettori in veste di testimoni di quanto sta mettendo in scena nel suo originale romanzo di formazione. Lo sfogo datato 1929, «Se si potesse, se si potesse ripudiare il proprio nome, invece di farne mercimonio per vivere, sotto prose caduche, di giornale!» (FolLI 2000, 112), disvela la portata politica dell'appello in seno all'opera. Benché Negri godesse di una rendita vitalizia conseguita grazie al conferimento del Premio Giannina Milli nel 1894, continuò ad esercitare l'amata professione di insegnante e a pubblicare racconti brevi sui feuilleton di varie 
riviste dell'epoca. Era questa una consuetudine che accomunava diverse scrittrici d'allora e che, come ricorda Robin Pickering-Iazzi, dava loro modo di pubblicare con una certa assiduità:

The importance for women of publishing short fiction on the terza pagina should not be underestimated. First of all, the economic benefits likely provided some women with the opportunity to become professional writers. It may also have functioned as a sign of independence, and certainly success, in view of the authority conferred on the institution of cultural page, signified also by its prestigious Elzevir type. ${ }^{14}$

Malgrado ciò, Negri sentiva questo spazio, votato principalmente alla pubblicazione di racconti femminili, come una prigionia piuttosto che come una forma di apertura alle scrittrici. Il cocente risentimento per la condizione di operaia sfruttata dal nascente sistema capitalistico del Nord Italia di cui è vittima la madre si dipana simmetricamente alla denuncia della condizione di scrittrice subordinata alle alte sfere letterarie palesemente patriarcali che controllano l'editoria italiana. Non è un caso che «ai canti dell'operaia Vittoria, scanditi sul respiro dei telai giù nella fabbrica, rispondono dalle stanzette verso il giardino del palazzo di via Roma i canti di Omero. La fanciulla è finalmente penetrata, sangue ed anima, nella poesia» (SM 52). La bambina sente su di sé la responsabilità del sacrificio della madre/stella mattutina - che esce di casa prestissimo senza far rumore, lavora instancabilmente per poterla mantenere agli studi e ha dovuto rinunciare a crescere il suo unico figlio maschio per motivi economici - e dell'eredità del suo narrare, la sera, a porte aperte, «con pause e chiaroscuri d'inconscia sapienza, scolpendo le figure del suo ricordo con pochi tratti essenziali, illuminando all'improvviso certe scene con vivissime luci istantanee, frescando alla brava quadri d'insieme: guidata da un istinto d'arte che ignora di possedere, e morirà ignorato con lei». (SM 69) (enfasi mia) La capacità di scolpire raccontando, di affrescare scene con pochi tratti essenziali, non morirà con lei se Dinin lascerà che l'Altra-la Vera dentro di sé raccolga quell'arte del tessere storie e creare spazi femminili comuni in cui ritrovarsi e narrare anche l'altra storia, quella che Gerda Lerner definisce «unrecorded past», ${ }^{15}$ volutamente dimenticata dalla storiografia ufficiale. Dinin dichiara che «di nulla e nessuno, alla fine, le importa; fuor che di spiegare sé a se medesima» ( $\mathrm{SM} \mathrm{78).} \mathrm{Ma} \mathrm{in}$ 
questa specularità, ella più che ritrovarsi si ricrea - letterariamente e storicamente, come il soggetto del dipinto Girl Before a Mirror di Picasso, in cui il maestro cubista, argomenta Drucker,

doubles the image, living the woman first the depiction as "real" and secondly the depiction as "image". The transformative process of his own practice is thus doubly coded through this split imagery. In the first level of illusion she has a graphic and pictorial character which includes the distinguishing marks of her identity [...]. In the second level of illusion, coded to be read as the symbolic within the frame of the symbolic (the image within the image), as the marked mediation of a reflection within the unmarked mediation of depiction, she exists as the drawn portrait within the image. [...] No attempt is made to duplicate one image with the other, instead they function as transformations of visual material within the image. (DRUCKER 1994, 116117)

La lunga citazione diventa quanto mai significativa se confrontata con le riflessioni di Dinin sull'Altra:

L'Altra: la Vera: che nessuno vedrà nel viso, nemmeno la mamma: inviolabile, inviolata: senza principio, senza fine: ricca d'inestinguibile calore al pari delle correnti sotterranee. Disgrazie, umiliazioni d'ogni sorta possono accadere alla pallida, povera Dinin; ma l'Altra, la Vera, è al di sopra di tutto e di tutti, è la Regina in incognito, che nulla può ledere. La sente, a volte, rivelarsi e sovrapporsi alla persona circoscritta respirante camminante, con la potenza d'un getto di lava; e ciò accade generalmente quand'ella, vagabondando sola, segue, lungo oscure straducole urbane, il suono degli organetti. (SM 88-89)

Dinin vagabonda per le strade urbane come una novella flâneuse baudelairiana in cui lo spazio che perlustra si dilata fino a diventare espace che le permetta di trasfigurarsi nell'Altra, nell'erede dei cantori poetici dell'antichità, nell'amazzone a cavallo degli endecasillabi foscoliani, nella compagna delle «immobili e portentose serenità del Leopardi» (SM 52). Se nello spazio di superficie del romanzo Dinin funziona come la ragazza ri-prodotta da Picasso nell'atto di specchiarsi, l'Altra non ne è il mero riflesso bensì l'immagine prodotta dalla scrittrice all'interno dell'espace che l'autrice/narratrice, il suo personaggio e il lettore concorrono a creare.

Quest'atto letterario può essere letto come una vera e propria presa di posizione politica, se si tiene conto delle dichiarazioni di Kristeva in Revolution in Poetic Language: 
If there exists a "discourse" which is not a mere depository of thin linguistic layers, an archive of structures, or the testimony of a withdrawn body, and is, instead, the essential element of a practice involving the sum of unconscious, subjective, and social relations in gestures of confrontation and appropriation, destruction and construction - productive violence, in short - it is "literature", or, more specifically, the text. [...] The text is a practice that could be compared to political revolution: the one brings about in the subject what the other introduces into society. ${ }^{16}$

Attivismo sociale e attività letteraria sono parti integranti dell'arte di Ada Negri, proprio come il personaggio Dinin - che subisce controvoglia le ingiustizie dell'ambiente in cui essa e le altre componenti della sua famiglia vivono - si muove specularmente al personaggio dell'Altra. È quest'ultima a legittimare la bambina a contravvenire ai dettami della società patriarcale ed a ricrearsi uno spazio alternativo in cui ci si possa esprimere attraverso una prospettiva linguistica che trascenda e travalichi ogni codificazione simbolica. Il processo di significazione, sostiene Kristeva, è il prodotto della continua oscillazione dialettica tra sfera simbolica e sfera semeotica e il senso che ne emerge rifrange l'io parlante in due entità complementari: soggetto che agisce e oggetto percepito dallo spazio sociale: «for Kristeva meaning is not the unified product of a unified subject; rather, meaning is Other and as such makes the subject other to itself. Meaning is not constituted by a transcendental ego; meaning is constituted within a biosocial situation $» .{ }^{17}$

La lingua che emerge dalla rifrazione del personaggio Dinin nell'Altra riecheggia le «parole senza suono, che son preghiere» (SM 13) di nonna Peppina; dipana i misteri dei «componimenti pieni d'inquietudini e di squilibrio, tralucenti d'immagini e di reminiscenze torbide e confuse» (SM 19) degli anni scolastici; la rimette in contatto con «[l]e cose [che] le sono vicinissime, trasparenti: [che] hanno occhi e respiro, [che] parlano il suo stesso linguaggio» (SM 23). Ecco allora Dinin-bambina che tende alla vita più che ai testi scolastici; che sogna di sottrarsi alla schiavitù della povertà, di rendersi libera, indipendente; ed ecco Dinin-figlia che legge tutto Zola «senza rimanerne lesa», perché «deve la figlia, tutto ascoltare, tutto vedere, tutto sapere» (SM 69). La agency dell'Altra trasforma Dinin nella testimone delle scrittrici semi-dimenticate dalla storiografia ufficiale: le mistiche del Duecento; le Petrarchiste; le letterate del Rinascimento e così via fino al ventesimo secolo. Il 'Giardino del Tempo', in cui 
ella si rifugia fin dalla più tenera età per dar sfogo alla sua vena poetica, si sovrappone al loro hortus conclusus e si apre finalmente verso l'esterno; guadagna spazio, anzi espace, permettendo loro di sconfinare dalla sfera privata, che per secoli le ha tenute prigioniere, ed accedere a quella pubblica, dominio prettamente maschile. Pickering-Iazzi rileva che

Negri's inventing of the mother-daughter relation as a vertical axis and thematic matrix [...] reconfigures the space of autobiography, breaking the patriarchal interdictions of culture and society. [...] Negri's articulation of the mother-poet may thus authorize interrelations between motherhood, self-recreation, and artistic creation. (PICKERING-IAZZI 1994, 68)

L'asse verticale madre-figlia funziona anche come continuazione ed esemplificazione dell'asse storico che lega le scrittrici tutte nella riformulazione dei parametri del genere autobiografico e, più specificamente, del Bildungsroman.

Nell'introduzione a Reading the Modernist Bildungsroman, Gregory Castle afferma che "the history of the Bildungsroman is the history of a genre in crisis» ${ }^{8}$ in quanto il modernismo ha più che mai messo in dubbio ogni certezza sull'idea di identità:

Modernism [...] can be regarded as a dynamic critical project in which aesthetic (especially literary) experimentation is directed at one of the most pressing concerns of the time: How do we define what is to be a human being? How do we acquire our selfhood, our identity? [...] the failure of the modernist Bildungsroman is a complex and contradictory phenomenon, for where the Bildungsheld (hero of the Bildung plot) fails to achieve inner culture or harmonious socialization, the genre itself appears to assert its integrity in powerful new ways, to exploit the formative and transformative power of failure in order to effect a rehabilitation of the Bildungsroman genre and a justification of the raison d'être of the form, Bildung. (CASTLE 2006, 1-2)19

Appare ovvio che la crisi non investa solo il genre ma anche e soprattutto il 'gender' nel caso delle scrittrici d'inizio Novecento in un'Italia sostanzialmente dominata dall'egemonia patriarcale - in cui Benedetto Croce non manca di accusare la stessa Negri di «rimanere chiusa nel suo 'io privato' mentre il poeta deve ritrovarsi 'solo con l'io universale'» (GoRINI SANTOLI 1995, 173). Essenzialmente apprezzata per la poesia, Ada Negri dedicò una parte solo marginale della propria fortunata carriera letteraria alla produzione prosastica, 
che tuttavia ebbe, come suggella Angela Gorini Santoli, «uno svolgimento copioso e straordinariamente multiforme» (GoRINI SANTOLI 1995, 116). Accanto ai volumi di racconti - in cui la psiche femminile ha immancabilmente il ruolo di protagonista e fulcro delle vicende principali - Stella mattutina indubbiamente rappresenta la creazione prosastica par excellence della scrittrice lodigiana. Eppure, nonostante la grande popolarità ottenuta dal volume già dalla prima edizione, e il quasi travolgente successo di pubblico sia entro i confini italiani che all'estero, dalle file dei maggiori letterati dell'epoca si levarono pochissime voci d'assenso nei confronti della scrittrice. Giosuè Carducci e Edmondo De Amicis furono tra i pochi acclamati letterati italiani ad esprimere un giudizio positivo sull'opera in questione. Il primo coraggiosamente loda la scrittrice definendola «Sana ed energica poetessa: Et erunt novissimi primi. Giacché ogni necessità, vigoria, precisione, verità vien sempre più mancando agli uomini decadenti e simbolisti, ben vengano le donne» (GoRINI SANTOLI 1995, 60). Al contrario, il tanto ammirato Giovanni Pascoli riservò a Negri critiche amare che la segnarono nel profondo, come dimostra la seguente dichiarazione dell'autrice:

Un lagno amarissimo di Giovanni Pascoli contro l'enorme diffusione dei miei primi volumi di versi [...]. Fra lui e me non passò che questo lagno carico di disistima per la mia povera opera poetica. Egli certamente aveva ragione. Ma che dovevo fare io? Tacere, ingoiare e continuare a scrivere come sentivo e come potevo. Naturalmente non ho mai cercato di conoscerlo; né gli ho mai mandato nulla di mio, come invece ho fatto con Gabriele D’Annunzio. Io porterò fino alla morte la dissonanza fra la smisurata popolarità che circonda la mia poesia e il suo reale e riconosciuto valore artistico: la porterò come una ferita che non si rimargina. Ma nessuno potrà mai accusarmi d'aver strisciato ai piedi dei rappresentanti della vera grandezza per illuminarmi del loro splendore. (Zurigo, 1914). (FolLI 2000, 113)

Se la citazione mette in luce il disappunto nel constatare l'accoglienza negativa delle sue opere da parte di un così importante scrittore nel panorama italiano, d'altro canto puntualizza la dignità con la quale Negri riconosce il proprio talento e si rifiuta di ridimensionarlo per non infastidire la vulgata dell'epoca, dichiaratamente sprezzante di un successo tutto al femminile. A tal proposito, non va sottovalutato il fatto che, fin dai suoi primissimi esordi letterari, la reputazione della scrittrice venne fortemente condizionata anche dal costante attivismo a favore delle donne e dei gruppi sociali più deboli. Oltre alla costante 
collaborazione con 'l'avvocato dei poveri' Luigi Majno (GoRINi SANTOLI 1995, 34) e la 'dottora dei poveri'º Anna Kuliscioff, la poetessa si adoperò in prima persona per fondare l'Unione femminile italiana, insieme ad Ersilia Majno, nonché la prima sede italiana del Club Soroptimist International, finalizzato alla solidarietà femminile tra Stati Uniti ed Europa. L'appellativo di 'vergine rossa' - che le sarebbe rimasto attaccato tutta la vita, trasformandosi in vera e propria nomea in occasione dello sfortunato attentato al presidente francese Carnot per mano di un anarchico compaesano della scrittrice, a lei sconosciuto - le deriva proprio dallo sfondo di denuncia sociale che percorre la sua intera produzione letteraria. La figura di letterata engagée filo-socialista a favore del popolo cozza prepotentemente con il consenso nei suoi confronti da parte del regime fascista e dello stesso Mussolini, che chiosò il successo di Stella mattutina con le seguenti parole: «Questo libro ci spiega come la prima attività poetica della Negri sia stata di carattere sociale. C'erano motivi intimi, oltre al colore del tempo. Il socialismo è stato per la Negri poetica, come per me, un'esperienza politica».21 $\mathrm{E}$ ancora, in un telegramma datato dicembre 1930, Mussolini scrive: «Ho letto oggi in un'ora di sosta il vostro volume Vespertina. [...] Voi avete toccato le corde più profonde della lirica italiana da Leopardi in poi. Avete realizzato una identità fra il vostro spirito e la varia vostra natura. Credete alla mia antica et immutabile devozione» (GORINI SANTOLI 1995, 99). Negri venne osannata dall'intellighenzia fascista perché sostanzialmente fraintesa. Il ritratto di una madre totalmente dedita al proprio lavoro di operaia, instancabile nonostante i massacranti turni di tredici ore e costretta a elemosinare il proprio stipendio in caso di infortunio, che campeggia in Stella mattutina, viene paradossalmente letto da Mussolini quale esempio di donna italiana votata al bene della famiglia e della patria - per le quali è pronta ad eclissarsi - piuttosto che vera e propria denuncia della disagiata condizione delle donne italiane, ridotte dal regime a mere produttrici di forza lavoro e madri di figli maschi. A quasi un secolo di distanza dalla pubblicazione, Stella mattutina sembra avere il peso di un vero e proprio manifesto politico: dall'espace lirico che fa da sostrato alla narrazione prosastica, Negri palesa il suo credo in un socialismo umanitario e in un'assoluta solidarietà femminile che poco 
hanno a che fare con l'ideologia marxista e ancor meno con le istanze fasciste. Il messaggio di denuncia che Ada Negri madre lascia all'amatissima figlia Bianca «A te Biancolina, gioia mia» recita la dedica sul frontespizio del romanzo - si sovrappone all'appello che Ada Negri scrittrice tramanda alle lettrici e letterate future. L'espace del romanzo è il terreno comune nel quale i destini femminili possono incrociarsi e concorrere a traslare una nuova concezione di genre e 'gender'. A bene vedere, la sua opera contribuisce in modo sostanziale alla lotta per le rivendicazioni femminili e alla revisione del canone letterario tradizionale in anticipo di quasi un decennio sull'uscita di A Room of One's Own, in cui

Virginia Woolf first brought the question of space to women's attention metonymically through the idea of 'a room of one's own', which came to represent the material circumstances women needed in order to be able to write, but space also lends itself metaphorically to highlighting women's relation to the canon and it is a metaphor that we meet over and over again in feminist criticism. [...] The writers achieve implicitly a profound re-working of the literary space itself as a relationship between reader and writer - as a new narrative space in dialogue with the old one. [...] the female writer's use of fantastic space offers an insight into her attitude towards the male-authored canon. (HIPKINS 2007, 5-6)

Le considerazioni di Hipkins sull'opera di Woolf mettono in luce la volontà della scrittrice inglese di ritagliarsi uno spazio al di fuori del canone maschile in ambito britannico che riecheggia la presa di posizione di Negri in territorio italiano, come giustamente enfatizzato da Pickering-Iazzi in The Politics fo Gender and Genre in Italian Women's Autobiography of the Interwar Years:

I will argue that Negri and Deledda write both within and against the conventions of formal autobiography - a genre which traditionally safeguards patriarchal relations of power by retelling the stories of manhood - and thus make an especially strong claim to the authority of female self-authorship. ${ }^{22}$

Se con la guerra - alla quale la scrittrice prende parte in prima persona come infermiera, al fianco dell'amatissima Bianca - l'antico sogno di un socialismo pacifista unitario crolla definitivamente, e se le sfortunate vicende amorose la portano ad una dolorosa disillusione verso l'unione di coppia, la vocazione alla poesia rimane vibrante in lei per tutta la vita. Alimentata dal costante riconoscimento di un pubblico internazionale, la sua volontà fu ferrea nell'allestire la sua opera letteraria come un vero e proprio palcoscenico dal quale 
rivolgere un appello che sensibilizzasse le scrittrici e lettrici del suo tempo e lasciasse in eredità uno spazio letterario - un espace letterario - alle future.

Essenzialmente votata alla poesia, si diceva, eppure solo all'interno della prosa infusa di lirismo di Stella mattutina Ada Negri riesce ad abbattere le barriere del 'gender' e a ridefinire i confini del genre del romanzo di formazione nel Novecento italiano. 
Note

1 Ada NEgRI, Stella mattutina, Milano, Otto/Novecento, 2008, p. 5. Da qui in poi SM.

2 Johanna DRUCKER, Theorizing Modernism: Visual Art and the Critical Tradition, New York, Columbia University Press, 1994, pp. 37-38.

3 Si fa riferimento in particolare a Kevin HETHERINGTON, Capitalism's Eye: Cultural Spaces of the Commodity, New York, Routledge, 2007 e a Aruna D'SouzA e Tom McDonough, The Invisible Flâneuse?: Gender, Public Space, and Visual Culture in Nineteenth-Century Paris, New York, Palgrave, 2006.

4 Angela Gorini SANTOLI, Invito alla lettura di Ada Negri, Milano, Mursia, 1995, p. 116.

5 Ada NEGRI, Tempeste, Milano, F.lli Treves, 1896, p. 308.

6 Ada NeGRI, Fatalità, Milano, F.lli Treves, 1900, p. 5.

7 A questo proposito è interessante riportare quanto rileva Ruth Shepard Phelps in An Italian Poetess: Ada Negri, in «The North American Review», Vol. 209, No. 761 (Apr., 1919), p. 529. «Ada Negri's early poems [...] were railing at no political oppressor, but at the more impersonal cruelties of the social order. [...] They [...] uttered the complaint of oppressed industry, which had never made itself heard in Italian verse before. They sang, in rather rough, stirring verses, the wretchedness of the helpless and the poor, of the old, and the beaten; they described the long miseries of unemployment, mutilated hands of factory women, the workman broken by his work, the vagabond who never knew a home and who lies at last beneath the stranger's dissecting knife, the bewildered family of evicted tenants, their humble goods confessing their paucity and pitiful domestic shifts too frankly to the daylight»

8 Anna Foldi, Penne leggere: Neera, Ada Negri, Sibilla Aleramo, scritture femminili italiane fra Otto e Novecento, Milano, Guerini, 2000, pp. 111-112.

9 Giorgio Rimond, Dall'Unheimlich all'oggetto, in Geografia Storia e Poetiche del Fantastico, di Monica Farnetti, Firenze, L. S. Olschki, 1995, p. 167.

10 Julia KRISTEVA, Language the Unknown - an Initiation into Linguistics, New York, Columbia University Press, 1989, p. 290.

11 Danielle HIPKINS, Contemporary Italian Women Writers and Traces of the Fantastic - The Creation of Literary Space, Leeds UK, Maney Publishing, 2009, pp. 2-3.

12 Liz Stanely, The Auto/biographical I - Theory and Practice of Feminist Auto/biography, New York, Manchester University Press, 1992, p. 61.

13 Monica FarnetTi, Geografia Storia e Poetiche del Fantastico, Firenze, Olschki Editore, 1995, p. 104.

14 Robin Pickering-IAZzI, Politics of the Visible, Minneapolis, University of Minnesota, 1997, p. 45 .

15 «What is history? We must distinguish between the unrecorded past - all the events of the past as recollected by human beings - and History - the recorded and interpreted past. Like men, women are and always have been actors and agents in history». Gerda LERNER, The Creation of Patriarchy, New York, Oxford University Press, 1986, p. 4.

16 Julia KristeVA, Revolution in Poetic Language, New York, Columbia University Press, 1984, p. 16.

17 Julia Kristeva, The Portable Kristeva, a c. di Kelly Oliver, New York, Columbia University Press, 2002, p. xviii.

18 Gregory CASTLE, Reading the Modernist Bildungsroman, Gainesville, University Press of Florida, 2006, pp. 1-2.

19 Castle sostiene inoltre che «the Bildungsroman is emblematic of certain tensions and contradictions within modernism, thus it is an exemplary genre for the representation of subjectivity, subject formation, and the relationship of the subject to modern social formations.», ibidem, p. 249.

20 Maricla Boggio, Anna Kuliscioff, Venezia, Marsilio, 1977, p. 110.

21 Salvatore Comes, Ada Negri da un tempo all'altro, Milano, Mondadori, 1970, p. 100.

22 Robin PICKERInG-IAZZI, The Politics of Gender and Genre in Italian Women's Autobiography of the Interwar Years, in «Italica», Vol. 71, No. 2, (Summer, 1994), p. 177. 


\section{Bibliografia}

\section{BOGGIO 1977}

Maricla BoggIO, Anna Kuliscioff, Venezia, Marsilio, 1977.

CASTLE 2006

Gregory CASTLE, Reading the Modernist Bildungsroman, Gainesville, University Press of Florida, 2006.

COMES 1970

Salvatore Comes, Ada Negri da un tempo all'altro, Milano, Mondadori, 1970.

D’SouZA e MCDONOUGH 2006

Aruna D’SouzA e Tom McDonough, The Invisible Flâneuse?: Gender, Public Space, and Visual Culture in Nineteenth-Century Paris, New York, Palgrave, 2006.

DRUCKER 1994

Johanna DRUCKER, Theorizing Modernism: Visual Art and the Critical Tradition, New York, Columbia University Press, 1994.

FARNETTI 1988

Monica FARNETTI, Il giuoco del maligno - il racconto fantastico nella letteratura italiana tra Otto e Novecento, Firenze, Vallecchi Editore, 1988.

FARNETTI 1995

Monica FARneTTI, Geografia Storia e Poetiche del Fantastico, Firenze, Olschki Editore, 1995.

FOLLI 2000

Anna Folli, Penne leggere: Neera, Ada Negri, Sibilla Aleramo, scritture femminili italiane fra Otto e Novecento, Milano, Guerini, 2000.

GORINI SANTOLI 1995

Angela GORINI SANTOLI, Invito alla lettura di Ada Negri, Milano, Mursia, 1995.

HETHERINGTON 2007

Kevin HeTHERINGTON, Capitalism's Eye: Cultural Spaces of the Commodity, New

York, Routledge, 2007.

HIPKINS 2007

Danielle HIPKINs, Contemporary Italian Women Writers and Traces of the Fantastic

- The Creation of Literary Space, Leeds UK, Maney Publishing, 2009.

KRISTEVA 1984

Julia KRISTEVA, Revolution in Poetic Language, New York, Columbia University Press, 1984. 


\section{KRISTEVA 1989}

Julia KRISTEVA, Language the Unknown - an Initiation into Linguistics, New York, Columbia University Press, 1989.

KRISTEVA 2002

Julia KRISTEVA, The Portable Kristeva, a c. di Kelly Oliver, New York, Columbia University Press, 2002.

LERNER 1986

Gerda LERnER, The Creation of Patriarchy, New York, Oxford University Press, 1986.

NEGRI 1896

Ada NEGRI, Tempeste, Milano, F.lli Treves, 1896.

NEGRI 1900

Ada NEGRI, Fatalità, Milano, F.lli Treves, 1900.

NEGRI 2008

Ada NEGRI, Stella mattutina, Milano, Otto/Novecento, 2008.

PICKERING-IAZZI 1994

Robin PICKERING-IAZZI, The Politics of Gender and Genre in Italian Women's Autobiography of the Interwar Years, in «Italica», Vol. 71, No. 2, (Summer, 1994).

PICKERING-IAZZI 1997

Robin PICKERING-IAZZI, Politics of the Visible, Minneapolis, University of Minnesota, 1997.

SHEPARD PHELPS 1919

Ruth ShePARD PhelPs, An Italian Poetess: Ada Negri, in «The North American Review», Vol. 209, No. 761 (Apr., 1919).

STANELY 1992

Liz STANELY, The Auto/biographical I - Theory and Practice of Feminist Auto/biography, New York, Manchester University Press, 1992. 